\title{
Influence of Mouse-Strain-Specific Factors on Position- Dependent Transgene DNA Methylation Patterns
}

\author{
P.A. Koetsier, W. Doerfler \\ Institute of Genetics, University of Cologne, Germany
}

In previous work from this laboratory, an inverse dependence was established for the adenovirus type $2 \mathrm{E} 2 \mathrm{~A}$ late promoter between sequence-specific DNA methylation and promoter activity [1-5; for reviews see ref. 6, 7]. The effect of DNA methylation on promoter activity was also assessed in the transgenic mice, which were obtained from microinjections of unmethylated or in vitro HpaII-premethylated pAd2E2AL-CAT DNA [1] into $\mathrm{F}_{2}$ zygotes from $\mathrm{B} 6 \mathrm{D} 2 \mathrm{~F}_{1}(\mathrm{C} 57 \mathrm{BL} / 6 \times \mathrm{DBA} / 2)$ hybrid mice. In CAT assays carried out on organ extracts from the pAd2E2AL-CAT mice, the inverse relationship was confirmed [2].

We studied the stability of the pAd2E2AL-CAT DNA methylation patterns in up to eight mouse generations and assessed the influence of the strain-specific genetic background. Three pAd2E2AL-CAT mouse lines were crossed with inbred DBA/2, C57BL/6 or $\mathrm{B} 6 \mathrm{D} 2 \mathrm{~F}_{1}$ mice. Parent-of-origin effects were controlled by exclusive hemizygous transgene transmission either via females or males. The founder animal of line 7-1 carried two groups of transgenes (A and B) on separate chromosomes. The transgene methylation patterns of the 7-1B transgenes and those of the lines 5-8 and 8-1 were stably transmitted.

Southern blot hybridization experiments $[8,9]$ revealed that the $7-1 \mathrm{~A}$ transgene methylation pattern was a cellular mosaic. In mixed-genetic-background offspring from 7-lA animals, $10 \%$ carried transgenes with HpaII-DNA methylation levels that were reduced from 40 to $10-15 \%$. This finding suggested that in this background the factors that supported high methylation levels were dominant. When inbred DBA/2 mice were the mates, $40 \%$ of the siblings carried demethylated transgenes, whereas this ratio amounted to only $10 \%$ in $\mathrm{C} 57 \mathrm{BL} / 6$ offspring (comparable to $\mathrm{B} 6 \mathrm{D} 2 \mathrm{~F}_{1}$ crossings). Transgene methylation patterns were not detectably influenced by the parent-of-origin.

Four DBA/2 offspring generations were analyzed beyond a 7-1A animal that carried demethylated transgenes. All offspring maintained the demethylated status and in some animals the transgenes had become even more demethylated. However, in this almost pure genetic background, a low level of transgene methylation remained, which was still 
cellular mosaic. Hence, the detected cellular mosaicism was presumably not the consequence of a mixed genetic background. Rather, the strain-dependent cellular mosaic methylation patterns of the 7-1A transgenes might be the consequence of a chromosomal position effect, since the 7-1B transgenes in the same founder animal and the transgenes in the other mouse lines did not show such patterns.

Transgene methylation patterns were identical in the DNAs from all organ systems that were analyzed, except the testis, where the transgene DNA appeared to be almost completely demethylated, and epididymal sperm DNA, where the transgenes were de novo methylated. These different methylation levels might be indicative of methylation reprogramming events that take place in the germ line. However, the sperm transgene methylation patterns did not serve as a template for the (demethylated) patterns found in the 7-1A offspring. A more detailed account of this work will be published elsewhere [9].

Acknowledgement: This work was supported by the Deutsche Forschungsgemeinschaft through SFB 274-A1 and by the Fritz-Thyssen-Stiftung, Köln.

\section{REFERENCES}

1. Langner KD, Weyer U, Doerfler W: Trans effect of the El region of adenoviruses on the expression of the procaryotic gene in mammalian cells: Resistance to 5'-CCGG-3' methylation. Proc Natl Acad Sci USA 1986; 83: 1598-1602.

2. Lettmann C, Schmitz B, Doerfler W: Persistence or loss of preimposed methylation patterns and de novo methylation of foreign DNA integrated in transgenic mice. Nucleic Acids Res 1991; 19: 7131-7137.

3. Muller U, Doerfler W: Fixation of the unmethylated or the 5'-CCGG-3' methylated adenovirus late promoter-cat gene construct in the genome of hamster cells: Gene expression and stability of methylation patterns. J Virol 1987; 61: 3710-3720.

4. Toth M, Lichtenberg U, Doerfler W: Genomic sequencing reveals a 5-methyl-cytosine free domain in active promoters and the spreading of preimposed methylation patterns. Proc Natl Acad Sci USA 1989; 86: 3728-3732.

5. Toth M, Müller U, Doerfler W: Establishment of de novo methylation patterns: Transcription factor binding and deoxycytidine methylation at $\mathrm{CpG}$ and non-CpG sequences in an integrated adenovirus promoter. J Mol Biol 1990; 214; 673-683.

6. Doerfler W: DNA methylation and gene activity. Annu Rev Biochem 1983; 52: 93-306.

7. Doerfler W: Adenoviral DNA integration and changes in DNA methylation patterns: different view of insertional mutagenesis. Prog Nucleic Acids Res Mol Biol 1993; 46: 1-36.

8. Koetsier PA, Schorr J, Doerfler W: A rapid optimized protocol for downward alkaline blotting of DNA. Biotechniques 1993; 15: 260-262.

9. Koetsier PA, Mangel L, Schmitz B, Doerfler W: Stability of transgene methylation patterns in mice: Position effects, strain specificity and cellular mosaicism. Transgenic Res, in press.

Correspondence: Walter Doerfler, Institut für Genetik, Universität zu Köln, Weyertal 121 - D-50931 Köln, Germany. E-mail: doerfler@scan.genetik.uni-koeln.de. 